\title{
Chapter 13 \\ The Path to Heavy Ions at LHC and Beyond
}

\author{
Hans H. Gutbrod
}

\begin{abstract}
My appreciation of Rolf Hagedorn motivates me to look back at my more than 40 years of trial and error in relativistic heavy ion physics. More than once, wise colleagues helped me move forward to new and better understandings. Rolf Hagedorn was one of these important people. At first, I met him anonymously in the mid 1970s when reading his 1971 Cargèse Lectures in Physics, and later in person for many years in and around CERN. I wonder what this modest person would say about his impact on physics in this millennium. As he is not here to answer, I and others give our answers in this book. I focus my report on the beginning of the research program with relativistic heavy ions, the move to CERN-SPS and the development of the heavy ion collaboration at the CERN-LHC.
\end{abstract}

\subsection{Work at the Bevalac}

For more than four decades, I have studied relativistic collisions of heavy nuclei with the goal to create matter at extreme density and temperature, as it may exist in Supernovae implosions, or in the Early Universe. It began in 1975, when I was working at the Lawrence Berkeley National Laboratory (LBL) Bevalac accelerator complex, using beams of $0.2-2 \mathrm{GeV} / \mathrm{c}$ medium heavy ions.

Would nuclei be dense enough to create a compressed fireball in relativistic nuclear collisions, or would they just pass through each other, producing nuclear shock waves in each other? In 1974, an experiment with $\mathrm{AgCl}$ detectors claimed to have seen the shock waves, although with very low statistics and little particle identification for the reaction products.

My group wanted to measure nuclear shock waves employing electronic detectors of many sorts, but we did not find them. Instead we found coalescence of nucleons forming light clusters due to high density in phase space of the collisions. The inclusive proton spectra looked very thermal. This led to the formulation of the

H.H. Gutbrod ( $\square)$

FAIR Russia Research Centre, NRC "Kurchatov Institute" FSBI "SSC RF ITEP",

B. Cheremushkinskaya, 25, Moscow, 117218, Russia

GSI, Helmholtzzentrum für Schwerionenphysik, Planckstrasse 1, 64291 Darmstadt, Germany

(C) The Author(s) 2016

J. Rafelski (ed.), Melting Hadrons, Boiling Quarks - From Hagedorn Temperature

to Ultra-Relativistic Heavy-Ion Collisions at CERN,

DOI 10.1007/978-3-319-17545-4_13 
nuclear fireball model, a very simple one. For a while our little model was the best in describing the experimental inclusive spectra.

Coming from low energy physics, the fireball was for me at first just a hot 'compound nucleus', but I learned quickly that at these relativistic energies particle and resonance production had to be included. This was when I 'met' Rolf Hagedorn through his 1971 Cargèse Lectures in Physics and that is how this story began. Our Bevalac collaboration drew the attention of Léon Van Hove, who was then director of CERN. In 1977, he visited me at LBL and listened to me, the youngster, explaining to him our experiment and our understanding at that time. I was proud to tell him that at the Bevalac we knew the work of a CERN theorist, named Rolf Hagedorn.

However, by 1977/1978, I was also very frustrated: We had not seen any experimental proof that heavy ion collisions were more than just an ensemble of nucleon-nucleon collisions, as described in intra-nuclear cascade models of that time. I recognized fairly quickly that our small detector system was only able to measure a fraction of the particles coming from one collisions and that we needed a $4 \pi$ coverage to measure each event fully to see possible collective phenomena in these nuclear collisions.

With beams of ions of mass up to 40, the measured signals of the first experiments were not much different from proton-proton collisions and an added value for the complexity and difficulties in the acceleration of heavy ions was not clear. Later, in Helium-Helium (mass $4+4$ ) collisions at the ISR collider at CERN, no new physics was discovered either. All beginnings are difficult but this situation was outright catastrophic.

Then I came on invitation of Johann Rafelski and Rolf Hagedorn in early 1978 to visit CERN. Johann, a young nuclear theorist educated in Walter Greiner's group in Frankfurt, worked at CERN with Rolf Hagedorn and under the tutelage of Leon van Hove, Maurice Jacob and John S Bell. I saw that he recognized the striking opportunity that the CERN system of accelerators offered to the field of relativistic heavy ion physics. He was also the first nuclear theorist I met who recognized the paradigm shift of the new quark-gluon plasma (QGP) physics, carrying with his ideas his teacher and mentor of statistical and thermal physics, Rolf Hagedorn.

Through his Frankfurt-GSI (the heavy ion laboratory in Darmstadt) connection, Johann heard of my work and wanted to learn more about the experimental potential of Bevalac and the results obtained in my first experiments. During this visit, I learned about Hagedorn-Rafelski's recent work, the theory of finite size hadron gas. I did not yet in full realize that a revolution of the established particle physics wisdom was in the making. It certainly lifted my morale to learn that someone in a major laboratory of particle physics was paying attention to work by a few nuclear experimentalists who were struggling to find anything interesting in their Bevalac data.

Not anything interesting? I should note that at that time one of our Bevalac experimental competitors did announce a large entropy production in these nuclear collisions, which would have meant production of some new phase of matter produced at these low energies. However, my group could resolve this entropy puzzle by our detection of large amount of light nuclear clusters like deuterons, 
tritons, $3 \mathrm{He}$, and $4 \mathrm{He}$, which lowered the entropy production to 'normal' values. So, yes, at that time, not interesting.

Fortunately, thanks to generous support from our division leader Rudolf Bock at our home institute at GSI Darmstadt, my Bevalac collaboration could move quickly, reaching new heights. From 1978 to 1981, I constructed the "Plastic Ball" experiment at the Bevalac, the first electronic detector in relativistic nuclear physics with $4 \pi$ coverage and particle identification. In 1982-1984, Bevalac employed beams of Niobium (mass 93) and later of Gold ions (mass 197) at fixed-target energies from $200 \mathrm{MeV}$ to $1 \mathrm{GeV}$ per nucleon.

We promptly discovered two nuclear matter flow phenomena in the emission pattern of these collisions: the 'side-splash' and the 'squeeze-out.' The side splash was in the reaction plane, whereas the squeeze out was perpendicular to it. Inelastic scattering of spectator particles determined the reaction plane. These discoveries gave evidence for collective phenomena in relativistic heavy ion collisions, which could not be explained by standard nucleon-nucleon cascades in the collision.

Today these phenomena are called directed flow ( $v 1)$ and elliptical flow ( $v 2)$. These flow phenomena support the assumption that in the collision zone the nuclear participants interact with each other, building up pressure and thus, one surmises, matter of high density and temperature has been formed. These experimental data allowed us to extract first information of the equation of state of nuclear matter when compressed to near 2-3 times nuclear densities. In these initial experiments at the Bevalac, the energy available was comparable to Supernovae explosions, although our collisions were also generating a lot of heat, and thus, we believe, the compressed nuclear matter in these nuclear collisions was much hotter than in most extreme Supernovae.

Furthermore, the pion, and strange hadron, kaon and lambda production changed in these heavy ion collisions compared to proton-proton collisions, pointing to an ongoing hadron-chemistry inside this hot matter. At low energies, nucleons play the dominant part in the collision, therefore one talks of a 'nuclear fireball'. Perhaps the key lesson learned in these experiments was that one needs very large, massive heavy ions to form a dense nuclear fireball.

These results had established the relativistic heavy ion physics as a field where nuclear collisions produced collective features not known from $p p$ collisions. Hot compressed matter had been discovered and it was clear that very heavy nuclei were needed to form and study its behaviour. These results opened the path to go to higher energies; that is, higher compression and temperatures. Only at a bit higher collision energies, Hagedorn and Rafelski suggested, quarks and gluons inside the nucleons start participating in the collision. This is how the actual push towards the experimental discovery of the QGP came to be-in my opinion this happened at CERN and one of the important forces was this theoretical work in the late 1970s.

Questions arose where and how to get heavy ion beams of much higher energies than at the Bevalac. We developed new ideas for accelerators at GSI, Darmstadt and at LBL, Berkeley. At Berkeley, we came up with the VENUS collider proposal, where head on collisions of $20+20 \mathrm{~A} \mathrm{GeV}$ gold beams would go to very high densities and temperatures. This project was cancelled when the ISABELLE 
$200+200 \mathrm{GeV} p+p$ collider made space for the SSC project, the superconducting super collider which itself fell victim of US-national politics. However at BNL, the ready circular tunnel plus superconductor cooling system were left for further use. This ultimately became the collider project RHIC in 1983.

While all this was going on, Rudolf Bock, Reinhard Stock and I proposed to the German government to build a synchrotron with $100 \mathrm{~T}$ meter rigidity (SIS 100) at GSI, similar in design and specifications to the AGS at Brookhaven and the PS at CERN. When looking back, one must say that fortunately for the field of relativistic heavy ions, our proposal was turned down and GSI built just a 'modern Bevalac'. I say fortunately since our physics objective, the discovery and study of QGP, meant that the SIS 100 was too small, and its demise meant that we were encouraged to seek elsewhere the required experimental capability.

\section{2 $\ldots$ and at the SPS}

With Reinhard Stock and 40 others I proposed a heavy ion program at the CERN accelerator complex. I offered to move our collaboration and our detector, the Plastic Ball, from LBL to CERN, and Reinhard Stock offered to bring his Bevalac Streamer Chamber group to CERN. In 1982 a memorandum of understanding was signed by the GSI, CERN and LBL to get heavy ions to CERN. The terms were that GSI promised to bring an electron cyclotron resonance (ECR) ion source, LBL a radio frequency quadrupole (RFQ) linear accelerator to the CERN site. Rudolf Bock (GSI), Herrmann Grunder (LBL), Reinhard Stock (Uni Marburg), and many others including myself, we proposed at first experiments with heavy ions at the CERN PS.

Our view was strongly supported by the director of CERN non-LEP research programs, Robert Klapisch, who, however, saw the higher energy potential of the SPS as much more adequate. A decision had to be made by CERN at the time that LEP program constraints were already putting the entire plan for the CERN heavy ion program at risk. In the end a miracle happened and we got heavy ions at the SPS. This was an excellent development, offering us access to the high energy range, and clearly separated our program from the AGS program at Brookhaven, which was proposed shorty after ours at CERN.

At the beginning of the SPS heavy ion program (with Oxygen and Sulphur beams) very few scientists expected to see similar matter flow features like at the Bevalac. Again the question was if nucleons in nuclei would be dense enough to allow the formation of a QGP. In fact quite a few thought that at SPS energies there would be transparency in nucleon flow, ruling out collective effects and the formation of a quark-gluon fireball. This thinking was of course not what Hagedorn and Rafelski saw as the most likely outcome of heavy ion collisions at SPS.

The first runs with Oxygen ions in SPS took place in Fall of 1986. One year later, we had Sulphur ions and more than 400 scientists participated in six experiments at CERN. There were several predictions for exciting physics discoveries: (a) Johann Rafelski's strangeness enhancement, for which initially three experiments 
(NA35, NA36 and WA85) were specifically equipped, (b) high temperatures, which my experimental collaboration WA80 took on to measure via direct photon spectroscopy, (c) J/psi suppression which NA 38 set out to measure. Experiment NA34 addressed spectra of dileptons, a signature of dense hadronic matter.

All experiments had to master measuring the global event character, like impact parameter, multiplicity of charged particles, transverse energy flow, etc., which some did only after many years of trial and error. Hope was that we should find in the QGP temperatures above 'Hagedorn's limiting temperature', recognized as the freeze-out temperature for hadrons from a cooling down QGP. In nearly all SPS experiments, Rolf Hagedorn's limiting temperature was found in various measured hadron spectra.

The strangeness QGP signature was particularly successful. Strangeness and more specifically strange antibaryons were recognized and developed by J. Rafelski, at times in collaborations involving R. Hagedorn, B. Muller, M. Danos, as the key to the QGP discovery. In the early years, both QGP and abundant strangeness, were very "exotic" topics. As an example, Johann's strangeness presentation was relegated to the "exotica" section of the LBL conference proceedings in 1983 in company of 'Anomalons', a long forgotten false discovery. With a strong experimental program and clear objectives at SPS, very strong and diverse evidence for QGP was discovered in study of strange hadrons, in particular strange antibaryons.

My WA93 collaboration discovered flow phenomena in $200 \mathrm{~A} \mathrm{GeV} \mathrm{S+Au} \mathrm{col-}$ lisions at a level of 5-10 times weaker than at the Bevalac. As mentioned earlier, this became then a real industry of $v 1$ and $v 2$ measurements, now extended to much higher orders.

My group's experimental series WA80/93/98 was keen to measure direct photons, developed exquisite technologies and methods to do so. Some predicted QGP-temperatures of up to $1 \mathrm{GeV}$ in early stages of the SPS collisions. In retrospect we can say that this was impossible, as this would require huge compressed energy densities and very short thermalisation times. This is indeed impossible to achieve even at the LHC. In our initial optimism we hoped to measure these extreme conditions, but were realistic enough to prepare for low thermal photon yields of $\gamma_{\text {thermal }} / \pi^{0}$ ratios of a few percent only. However, the extreme values never showed up, and we could only measure an upper limit for thermal photons from a plasma with a temperature of about $220 \mathrm{MeV}$. At RHIC, our WA98 photon spectrometer was employed again and could measure indeed direct photons telling a temperature of about $280 \mathrm{MeV}$ of the QGP.

\subsection{How Heavy Ions Got into LHC and the ALICE Was Born}

I must admit that I like to create and build new things when physics asks for it and no other existing device can be scavenged or reused. So, as the research program at SPS evolved towards the announcement of the QGP discovery, some of my 
interests were already focused on future opportunities. As always, chance helps at the beginning: In 1983 at the relativistic heavy ion meeting at Brookhaven, I discussed with Carlo Rubbia topics of the future heavy ion collider at BNL, later called RHIC, when he told me: 'You will get your collider at CERN, with enough energy for your physics case'. During all this discussion we were walking very swiftly back and forth through the corridors of BNL physics department, as swiftly as Carlo's thoughts were rushing in.

At first, I did not understand what he was talking about. He argued that the quark physics signals became much clearer at the high energies of the Spp $\mathrm{S}$ collider compared to the smaller collider at CERN, the ISR. He kept his promise once he was Director of CERN and installed the heavy ion option into the LHC project from early on, among many things by insisting on a two-in-one magnet solution for the LHC allowing matter on matter collisions instead of a cheaper $p \bar{p}$ mode that is matter-antimatter beams sharing only one vacuum chamber.

Fast forward to the Aachen meeting in 1990: a small group (Ch. Fabian, H. Gutbrod, H. Specht, W. Willis et al.) sketched a detector concept comprising a large solenoid, coupled with one dipole at each end with full particle tracking. This was similar to the smaller $4 \pi$ detector the group had proposed several years earlier for the BNL collider.

From 1991 on, a small group of initially about 20 persons met at CERN regularly to work on a proposal for a dedicated heavy ion experiment at the LHC. In parallel we had to build and run our lead beam experiments at the SPS. From the start of discussing the concept of a dedicated heavy ion experiment at the LHC we had two concepts, one having a silicon tracker inside of a superconducting thin solenoid, pushed by Jürgen Schukraft, and one having all detectors inside of a large solenoid, pushed by myself.

Our consensus was that the large magnet solution required a much larger budget, but that the small magnet risked producing unwanted tracks in the detectors in the outside field free region with worse resolution due to multiple scattering in the magnet material. I must add further, that we had little guidance about the multiplicity of produced particles. Theoretical predictions ran from $d N / d y=2,000$ to 8,000 charged particles and of course as history has shown these as function of energy and collision centrality were only a bit off the actual result. So we needed to be prepared for the worst, ten thousand particles to be recognized and identified. I did not hesitate to push for the more expensive solution: At Berkeley I learned that one had to make adequate investments on the experimental side in order to make proper use of the costly beam time of the accelerator system itself.

At the LHC meeting at Evian-les-Bains in March of 1992, Jürgen Schukraft presented the small magnet project. The omission of the big magnet solution was due to the simple fact that there was no money to create a big detector from scratch. In the following months, I tried to find a cost effective solution: seeking a large magnet I looked first at the DEPLPHI solenoid magnet, which was unfortunately too small for the track density we anticipated in nuclear collisions.

Then, one late Spring day in 1992 in the CERN cafeteria when having lunch with Johann Rafelski I outlined to him in the presence of Paulo Giubellino and Lars 
Leistam and a few others what I saw as the real opportunity, the implementation of heavy ions in the L3 experiment. Its huge magnet was perfect for our purpose. At that time Sam Ting was proposing his own L3P experiment, i.e. an upgraded L3 setup for the LHC program. It was to me obvious to name my proposed set-up L3 H.I., hoping to interest the L3 team in the H.I. program from the beginning.

On July 2nd 1992, in one of our 'H.I. at LHC' proto-collaboration meetings at CERN, the question was raised: Should we have two parallel simulations, one for the small magnet and one for the large magnet? The decision was 'yes', we should follow both paths, and I was preparing the large magnet project, interacting with L3. Sam Ting asked me to present this proposal only after a decision was clear about L3P. In the Fall of 1992, the fate of L3P was clear, and Sam invited me to give a talk to his collaboration. I started my presentation saying: 'I feel somewhat awkward coming here wanting to steal your beautiful experiment.' From then on we got strong support from the L3 team as well as from the CERN management.

On February 1, 1993 the small magnet scenario was dropped and the L3 H.I. concept was adopted. The transfer of ownership of the L3 magnet and the infrastructure of the L3 site from the L3 collaboration to the H.I. collaboration was performed at a seafood dinner hosted by Jürgen Schukraft in a restaurant at Ferney-Voltaire and in March 1993, the Letter of Intent for 'A Large Ion Collider Experiment' (ALICE, see Fig. 13.1) was submitted as CERN/LHCC/93-

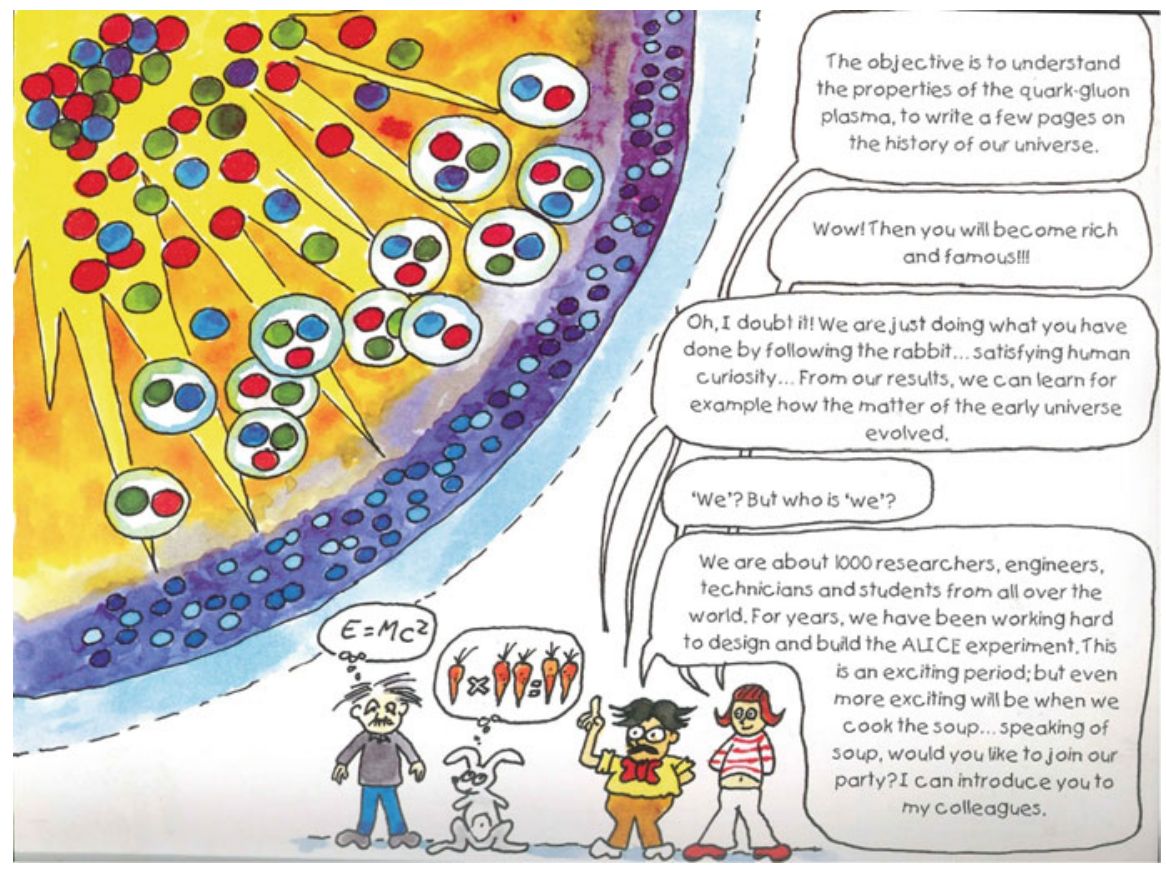

Fig. 13.1 ALICE at CERN explains itself in the 2004 outreach brochure-the backpage. Cartoonist: Jordi Boixader; Scenario and text: Federico Antinori, Hans de Groot, Catherine Decasse, Yiota Foka, Yves Schutz and Christine Vanoli 
16-LHCC/I4 on the basis of L3 H.I.. Jürgen Schukraft pushed it ahead towards completion and to many years of successful operation with striking new results and discoveries, published in a large number of publications.

One must note with delight, that the heavy ion physics at LHC has seen a tremendous strengthening due to the successful participation of the two $p p$ experiments ATLAS and CMS in the heavy ion runs. Competition in science is always good!

\subsection{Future Facilities}

Complementary to the scientific programs at RHIC and LHC, where the thrust is to create a fireball with vanishing baryon chemical potential, a new scientific push has been undertaken to study in detail the equation of state of highly compressed baryon-rich matter. In highly compressed 'cold' nuclear matter-as it may exist in the interior of neutron stars - the baryons lose their identity and dissolve into quarks and gluons. The critical density at which this transition occurs, however, is not known. The same is true for the entire high-density area of the phase diagram. At very high densities and low temperatures, beyond the deconfinement transition, a new phase is expected: the quarks are correlated and form a color superconductor. At the "critical point" the deconfinement/chiral phase transition is predicted to change its character.

I can say that Rolf Hagedorn's work on dense nuclear and hadron matter is pursued today with more vigor than ever before. Starting about 3 years ago, two new facilities have been under construction, and we are witnessing renewed interest in this energy range at CERN-SPS:

- At Darmstadt, Germany, the international FAIR facility is focusing on hadrons in compressed baryonic matter in the CBM experiment, and on hadron structures in proton-anti-proton reactions in the PANDA experiment. High precision measurements will allow the determination of the lifetime and mass of hadrons to new precisions. (Additional FAIR scientific programs are in nuclear structure and astrophysics, atomic physics, plasma physics and applied physics. I had the pleasure to lead this project until 2008.)

- At Dubna, Russia, the project NICA is going to study baryon rich systems in a fixed target experiment BMN and in an ion collider experiment MPD at NICA.

- At CERN SPS a pilot program uses the full available low energy range exploring the discovery potential of the above two new experimental facilities.

- We recall the AGS at BNL before RHIC came on-line nearly reached this energy domain, albeit with limited instrumental detector capacity and very limited beam time.

The heavy-ion collisions at FAIR and NICA energies permit the exploration of the "terra incognita" of the QCD phase diagram in the region of high baryon densities. At both facilities FAIR and NICA, fully dedicated research with nuclear 
collisions of highest intensities will allow us to create highest baryon densities, to explore the properties of super-dense nuclear matter, to search for in-medium modification of hadrons, the confinement of quarks in hadrons, to shed light on the restoration of chiral symmetry, to give insight into the origin of hadron masses, to look for the transition from dense hadronic matter to quark-gluon matter, and for the critical endpoint in the phase diagram of strongly interacting matter, and finally to provide 'hopefully' understanding of the structure of neutron stars and the dynamics of core-collapse supernovae.

\subsection{Epilogue}

In 1993/1994 I worked with Johann Rafelski to organize in Divonne, not too far from CERN, the fest to celebrate Hagedorn's 75th Birthday (Figs. 13.2 and 13.3), with his consent. Johann invited me to join forces with the otherwise theoretical team he formed with other friends and admirers of Hagedorn: Steven Frautschi, Jean Letessier and Helmut Satz. He explained, he wants the meeting and the dedicated book volume to have the subtitle "Theory and Experiment" in the spirit of Hagedorn. After a splendid celebration week at the end of June 1994, we worked together to publish 'Hot Hadronic Matter', a thick 550 pages volume dedicated to Rolf Hagedorn with the cornerstone observables discussed in depth. In fact the first extensive discussion of the design of the LHC ALICE experiment is presented in this volume on my behest.

I have spent the last 20 years at three laboratories directing, leading, and building many instruments that today form the backbone of the world-wide effort to study and explore the QGP phase of matter. I have seen the first step of the heavy ion program of research at CERN completed with the announcement of the discovery of the new state of matter in early 2000. As predicted by Carlo Rubbia, the high

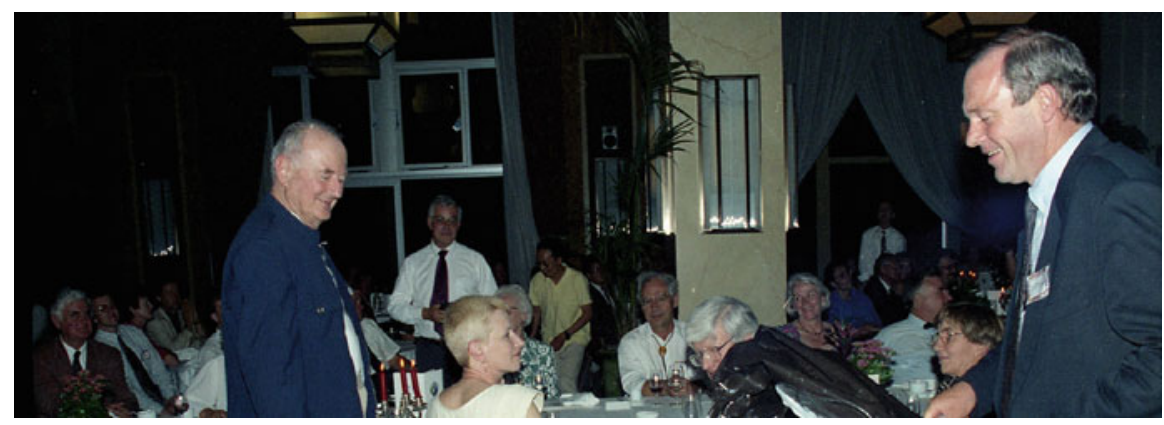

Fig. 13.2 Forefront Standing: left Rolf Hagedorn received his 75th anniversary gift from the organizers of the Divonne Conference, represented by Hans H. Gutbrod standing on right. Next to Hagedorn's right standing Maurice Jacob. Left of Hagedorn sitting at image edge: Luigi Sertorio, Image credit: CERN Image 199406-067-014 


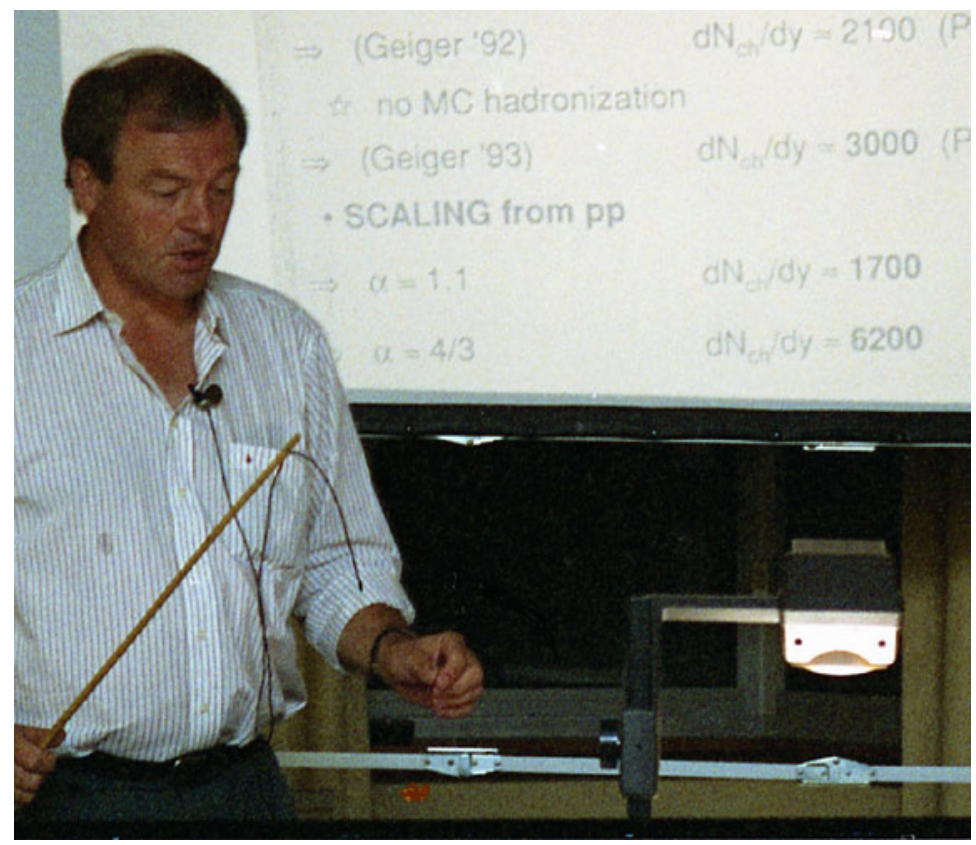

Fig. 13.3 Hans Gutbrod lecturing at Divonne on future high energy collider $A A$ experiments. We note on the screen the results of Klaus Geiger, see Fig. 14.2. Credit: CERN Image 1994-06-068-008

energies at LHC have given through ALICE, ATLAS and CMS, deep insight into this new deconfined phase of matter.

Every year exciting results appear due to instrumental advances: We gain a greater capability to observe and evaluate the large and diverse particle multiplicity. Thus we can address today more precisely and convincingly the established observables of the plasma phase and in doing this we begin to understand plasma properties, evolution history, and the mechanisms governing the hadronization process. We seek also to understand in detail the thresholds in volume size and energy that govern formation of the new deconfined state of matter.

It is abundantly clear to me that the program of research as it is constructed and executed presently relies on principles and ideas that were recognized in the first years at CERN, when the SPS program was developed, and which relies in a great measure on the legacy of Rolf Hagedorn. It is a great pleasure for me to have contributed a little bit to establishing a future for young scientists to go much further than we could do in the past. Rolf Hagedorn has built the base for this physics.

Open Access This book is distributed under the terms of the Creative Commons Attribution Noncommercial License which permits any noncommercial use, distribution, and reproduction in any medium, provided the original author(s) and sources are credited. 